\title{
Le rôle de la phase hépatique dans la protection antipaludique
}

La protection induite par immunisation avec des sporozoïtes irradiés a été à l'origine d'un espoir concernant la mise au point d'un vaccin antipaludique. En premier lieu attribuée aux seuls anticorps antisporozoïtes, cette immunité protectrice est en fait la résultante de mécanismes effecteurs complexes et intriqués. Anticorps, cytotoxicité restreinte par le CMH ou dépendante des anticorps, libération de cytokines (interférons, interleukines, TNF) et phénomènes inflammatoires semblent tous impliqués.

\section{Dominique Mazier}

\section{ADRESSE}

D. Mazier: maître de conférences. Inserm U. 313 et département de parasitologie, groupe hospitalier Pitié-Salpêtrière, 91, bou- a recherche sur la mise au point d'un vaccin antipaludique concernait jusqu'à présent trois cibles, le sporozoïte, le stade érythrocytaire, le gamète (figure 1, flèches 1, 2 et 3 ). Les potentialités du stade hépatique (figure 1 , flèche 4) sont restées presque totalement inexplorées. Les raisons en sont multiples : découverte tardive, presque cinquante ans après celle des autres stades, absence de manifestations cliniques, problème d'obtention du matériel d'étude en quantité suffisante, difficultés d'investigation chez l'homme pour un parasite localisé dans le foie, enfin et surtout, manque d'intérêt pour un parasite intracellulaire supposé être inaccessible au système immunologique. La mise au point de cultures [1] et l'utilisation de singes réceptifs aux plasmodies humaines, au moins au niveau hépatique [2], ont modifié cette situation. Longtemps négligée, la phase hépatique suscite maintenant un intérêt croissant. Le rôle qu'elle joue apparaît en effet primordial dans l'induction d'une protection dirigée contre les formes pré-érythrocytaires. Il est clair que l'interdépendance est grande entre stade hépatique et sporozoïte, le schizonte hépatique étant la cible de mécanismes effecteurs induits par le sporozoïte [3]. Des travaux récents démontrent qu'une telle interdépendance pourrait également exister entre stade hépatique et stade érythrocytaire. Ainsi, à côté d'un intérêt potentiel en tant que cible antigénique originale [4], le stade hépatique pourrait jouer un rôle crucial comme intermédiaire privilégié entre les autres stades [5].

Obtenir une protection contre les 


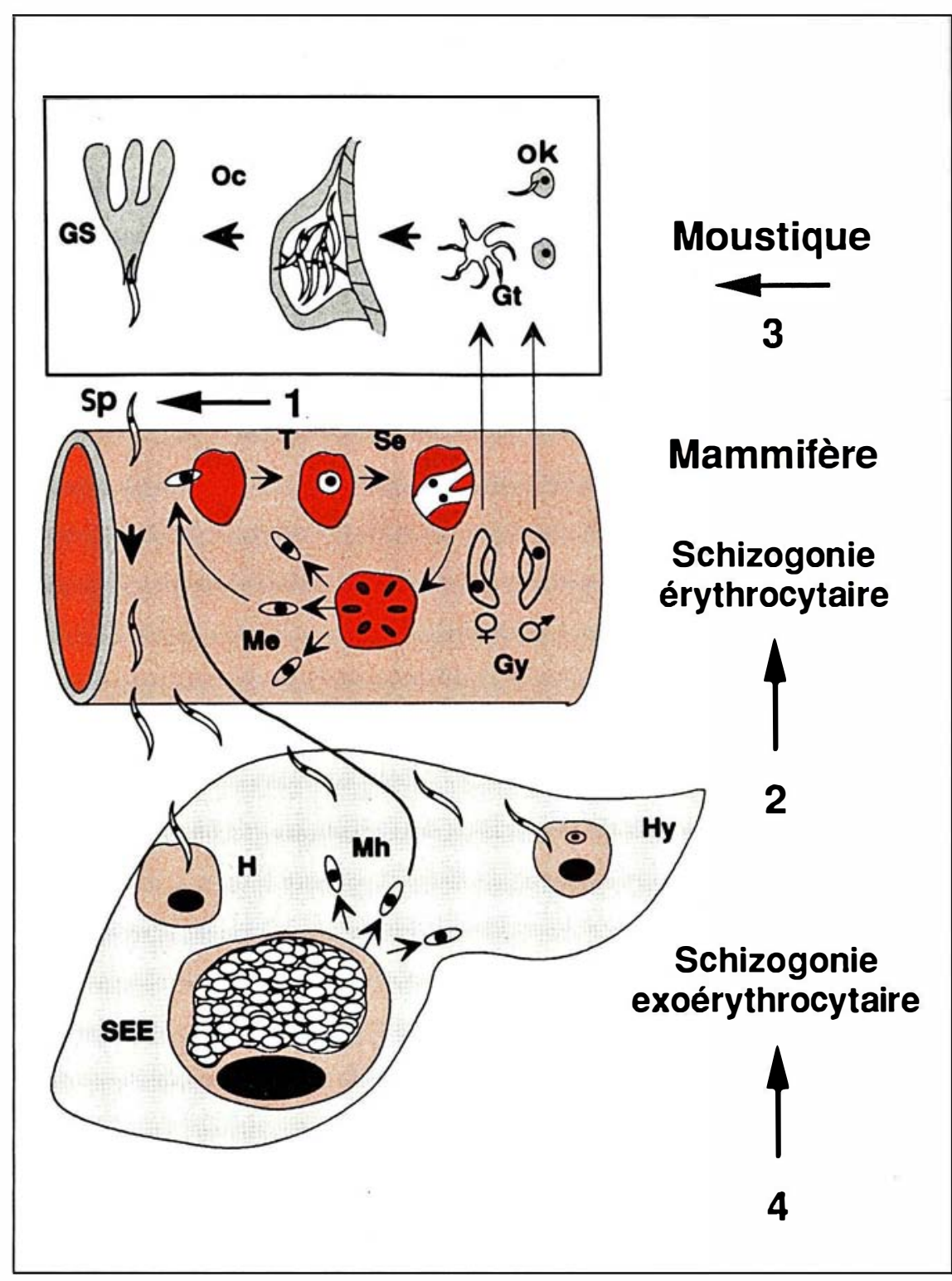

stades pré-érythrocytaires est possible, comme le prouve la résistance observée chez les souris [6] et les hommes [7] immunisés avec des sporozoïtes irradiés. L'objectif d'un vaccin antisporozoïte serait de mimer cette puissante réponse de protection. Cette immunité protectrice a été en premier lieu attribuée aux anticorps dirigés contre la partie répétitive de la protéine circumsporozoïte (CS), protéine majeure de la surface du sporozoïte [8]. Les essais de vaccination fondés sur l'induction d'anticorps anti-CS n'ont cependant eu qu'un succès limité, chez l'homme $[9,10]$ comme chez la sou$\mathrm{m} / \mathrm{s} n^{\circ} 10$ vol. 5 , décembre 89
Figure 1. Cibles vaccinales. Aux modifications morphologiques du parasite tout au long de son cycle, correspondent des modifications antigéniques responsables de réponses immunitaires différentes. On peut ainsi individualiser quatre cibles, chacune ayant ses caractéristiques propres.

1. Le sporozoïte. Classiquement, le vaccin anti-sporozoïte se doit d'être parfait, un seul sporozoïte pouvant théoriquement donner naissance à un schizonte hépatique à l'origine de la libération de milliers de mérozoïtes infectieux. Cet absolutisme parait trop pessimiste: l'arrivée non massive de mérozoïtes d'origine hépatique et l'installation lente d'une parasitémie semble permettre à l'organisme la maîtrise du développement parasitaire.

2. Le stade érythrocytaire. L'ambition d'un vaccin dirigé contre ce stade n'est pas la stérilisation parasitaire complète. Un reliquat de parasitémie, persistant à taux très faibles, n'engendrant pas de manifestations cliniques majeures, mimant ainsi ce qui s'observe en zone d'endémie palustre, serait tout à fait acceptable.

3. Le gamète. Vaccin "altruiste", car ne protégeant pas l'individu vacciné, il trouve sa place dans les campagnes de masse, en réduisant la transmission. En effet l'absorption, par le moustique piquant un sujet vacciné, d'anticorps antigamètes, empêche la fécondation des formes sexuées et donc la production de sporozoïtes par ce même moustique.

4. Le stade hépatique. Les potentialités d'une telle cible sont restées longtemps inexplorées; il est maintenant admis que son intérêt est double : (1) en tant que cible antigénique spécifique, (2) en tant que cible de mécanismes effecteurs induits par les autres stades. Le cloisonnement par cible apparait ainsi artificiel. La spécificité de stade n'est pas aussi stricte que ce que l'on a longtemps pensé, mais surtout l'importance des interactions et des rétroactions entre les stades pose le problème du type de test qu'il faut effectuer pour vérifier la valeur d'une vaccination. révu, contrairement aux mécanismes cellulaires qui apparaissen maintenant comme primordiaux. Plusieurs observations sont en accord avec cette hypothèse: (1) l'immunisation par des sporozoïtes irradiés est efficace même chez des souris déplétées en lymphocytes $B$ [12]; (2) des cellules T suppressives/cytotoxiques exprimant le phénotype $\mathrm{CD}^{+}$participent au développement d'une immunité stérile[13,14]; (3) l'administration orale de Salmonella typhimurium, transformée par le gène de la CS de $P$. Ber-
Abréviations. $G S=$ glandes salivaires; $S p=$ sporozoite ; $H=$ hépatocyte; $H y=$ hypnozoïte; SEE = schizonte exoérythrocytaire; $M h=$ mérozoïte hépatique; $T=$ trophozoïte $\mathrm{Se}=$ schizonte érythrocytaire; $\mathrm{Me}=$ mérozoïte érythrocytaire ; Gy = gamétocyte $; G t=$ gamète $; O K=$ ookinète $; O c$ =oocyste. 


\section{RÉFÉRENCES}

1. Mazier D. Use of cultured hepatocytes in parasitology. In : Guillouzo A, Guguen-Guillouzo C, eds. Research in isolated and cultured hepatocytes. Paris: John Libbey Eurotext/ INSERM, $1986:$ 378-97.

2. Druilhe $P$, Miltgen F, Landau I, Rinjard J, Gentilini M. Schizogonie hépatique de Plasmodium falciparum chez le singe Cebus apella. CR Acad Sci Paris 1982 ; 294 : 511 -3.

3. Mazier D, Miltgen F, Nudelman S, et al. Pre-crythrocytic stages of plasmodia. Role of specific and non specific factors. Biol Cell $1988 ; 64$ : 165-72.

4. Guerin-Marchand C, Druilhe P, Galley B, et al. A liver-stage-specific antigen Plasmodium falciparum characterized by gene cloning. Nature 1987 ; 329 : 164-7.

5. Mazier D, Goma J, Pied S, et al. Hepatic phase of malaria. A crucial role as «gobetween " with other stages. Am J Trop Med Hyg 1990 (sous presse)

6. Nussenzweig RS, Vanderberg J, Most $\mathrm{H}$ Orton C. Protective immunity produced by injection of X-irradiated sporozoites of Plasmodium berghei. Nature 1967 ; 216 : 160-2.

7. Reickmann RH, Carson PE, Beaudoin RL, Cassells JS, Sell KW. Sporozoite induced immunity in man against an Ethiopian strain of Plasmodium falciparum. Trans $R$ Soc Trop Med Hyg 1974; 62 : 258-9.

8. Nussenzweig V, Nussenzweig RS. Circumsporozoite proteins of malaria parasites. Cell 1985 ; 42 : $401-3$.

9. Ballou WR, Sherwood JA, Neva FA, et al. Safety and efficacy of a recombinant DNA Plasmodium falciparum sporozoite vaccine. Lancet 1987 ; 8545 : 1277-81.

10. Herrington DA, Clyde DF, Losonsky G, et al. Safety and immunogenicity in man of a synthetic peptide malaria vaccine against Plasmodium falciparum sporozoites. Nature $1987 ; 328: 257-63$

11. Egan JE, Weber JL, Ballou WR, et al. Efficacy of murine malaria sporozoite vaccines : implications for human vaccine development. Science 1987 ; 236 : 453-6.

12. Chen DT, Tigelaar RE, Weinbaum FI. Immunity to sporozoite-induced malaria infection in mice. 1 . The effect of immunization of $\mathrm{T}$ and B cell-deficient mice. J Immunol 1977 ; 118 : 1322-7.

13. Schofield L, Villaquiran J, Ferreira A, Schellekens H, Nussenzweig RS, Nussenzweig V. Gamma interferon, $\mathrm{CD}^{+} \mathrm{T}$ cells and antibodies required for immunity to malaria sporozoites. Nature 1987 ; 330 : 6646 ghei, induit chez la souris une immunité cellulaire spécifique de la CS et une protection malgré l'absence d'anticorps anti-sporozoïte [15].

Les mécanismes induits au cours d'une immunisation avec des sporozoïtes irradiés sont cependant loin d'être élucidés. Infiniment plus nombreux et intriqués que prévu, ils mettent en jeu anticorps, mécanismes cellulaires et facteurs non spécifiques.

\section{Anticorps anti- sporozoite : effets biologiques opposés}

Le rôle primordial attribué en premier lieu aux anticorps anti-sporozoïte a conduit à de très nombreuses expérimentations in vitro, à l'aide de primocultures d'hépatocytes humains [16-18] ou murins [19] ( $f i$ gure 2). Ces tests ont clairement montré que, quel que soit leur type (polyclonal ou monoclonal) ou leur origine (naturelle ou consécutive à une immunisation avec peptides synthétiques ou recombinants), les anticorps anti-CS avaient en commun plusieurs propriétés.

- Ils inhibent la pénétration du sporozoïte dans l'hépatocyte $[18,20$, 21].

- Cette inhibition n'est que rarement complète. Quelques sporozoïtes échappent à l'action des anticorps, et cela quelle que soit la concentration de ces anticorps [22].

- Leur activité n'est pas limitée à la phase de pénétration. L'attachement du sporozoïte à la membrane hépatocytaire et, de façon plus surprenante, le développement intrahépatique du parasite sont modifiés par les anticorps anti-CS. Cet effet "postpénétration » a d'abord été observé avec P. falciparum [20]. Un même phénomène a été plus récemment décrit avec $P$. yoelii [21]. L'utilisation d'une technique de double marquage qui permet une analyse précise des différentes étapes de la pénétration et du développement du sporozoïte [23] nous a permis de «décortiquer» le mécanisme. Les anticorps anti-CS poursuivent leur activité sur le stade suivant, le trophozoïte, dont ils entraînent l'arrêt du développement, souvent même la disparition. La destruction du parasite intracellulaire est en relation avec la présence des anticorps anti-CS dans la vacuole parasitophore, où ils sont visualisables par des anti-IgG couplés à la fluorescéine. La protéine CS persistant tout au long de la schizogonie hépatique [1], les anticorps anti-CS liés au parasite agiraient en bloquant la translocation de la protéine CS à la membrane de la vacuole parasitophore. Ce phénomène, en augmentant l'acidité à l'intérieur de la vacuole parasitophore, induirait, comme dans le cas de Toxoplasma gondii [24], la digestion lysosomique du trophozoïte.

L'intérêt d'un tel effet intracellulaire dépasse le fait qu'il améliore une activité a priori considérée comme devant être restreinte à la pénétration du sporozoïte. Cet effet n'est pas limité à l'action des anticorps. Nous verrons plus loin qu'une activité comparable est observée avec la protéine C-réactive (C-reactive protein, CRP). Dans tous les cas, un mécanisme similaire est impliqué : incorporation de molécules, anticorps ou non, au moment où la vacuole parasitophore est créée par invagination de la membrane hépatocytaire durant l'invasion du sporozoïte; nous avons antérieurement mentionné [18] qu'un point critique dans le développement hépatique consistait en l'initiation de la première division nucléaire. La translocation de la protéine CS à la membrane de la vacuole parasitophore pourrait être une étape cruciale de cette initiation. L'impossibilité, pour la protéine CS de $P$. falciparum, de transloquer dans l'hépatome Hep- $\mathrm{G}_{2}$, a été corrélée à l'incapacité pour cette lignée cellulaire de permettre le développement de cette plasmodie [25] La compréhension des mécanismes, positifs ou négatifs, présents dans les hépatocytes humains sains et non dans l'hépatome, serait évidemment du plus grand intérêt.

- Ils facilitent la pénétration du sporozoïte dans l'hépatocyte [21]. Des expériences réalisées avec des dilutions sériées montrent qu'à titre faible, les anticorps dirigés contre la protéine CS sont capables, in vitro, d'augmenter le nombre de parasites hépatiques jusqu'à $150 \%$ du nombre obtenu dans les contrôles. L'importance de ce phénomène, observé avec 
des anticorps polyclonaux et monoclonaux (IgG et IgM), est inversement proportionnel à la capacité inhibitrice de l'anticorps. Le phénomène a lieu durant les premières étapes de la pénétration du sporozoïte et résulte non pas d'un effet direct sur le sporozoïte, mais d'une modification dans l'interaction entre sporozoïte et membrane hépatocytaire. Décrits chez les virus [26] ou le parasite Toxoplasma gondii [27], les phénomènes de facilitation ont été récemment observés dans le cas du Plasmodium, aux stades gamète [28] et sporozoïte mais durant la phase anophélienne [29]. L'hypothèse initiale supposait, comme dans les modèles viraux, une médiation par les récepteurs Fc [30] ou les récepteurs au complément [31]. Le fait que cette facilitation soit également observée avec des fragments monovalents Fab plaide contre cette première alternative. Il est plus vraisemblable que nous ayons affaire à une modification de conformation de la protéine CS, cette modification donnant naissance à une structure capable de se lier plus efficacement au récepteur présomptif. Si ces observations faites in vitro se vérifient in vivo, la facilitation induite par les anticorps pourrait être un phénomène important à prendre en compte dans la mise en place des futurs vaccins antisporozoïte. De nombreuses questions fondamentales concernant la pénétration du sporozoïte restent en suspens. En tout état de cause, la compréhension des mécanismes de facilitation pourrait en partie permettre de mieux comprendre cette étape.

\section{Mécanismes cellulaires}

Cytokines : mode d'action direct ou indirect. Après l'engouement suscité par les anticorps, la tendance actuelle est d'attribuer un rôle essentiel aux mécanismes cellulaires. De nombreuses cytokines interfèrent avec le Plasmodium durant sa phase hépatique, directement et/ou dans le contexte d'interactions complexes, avec la participation de nombreuses cellules incluant l'hépatocyte luimême.

- Interféron $\gamma$. Parmi les diverses cytokines relarguées par les lympho-

$\mathrm{m} / \mathrm{s} n^{\circ} 10$ vol. 5 , décembre 89

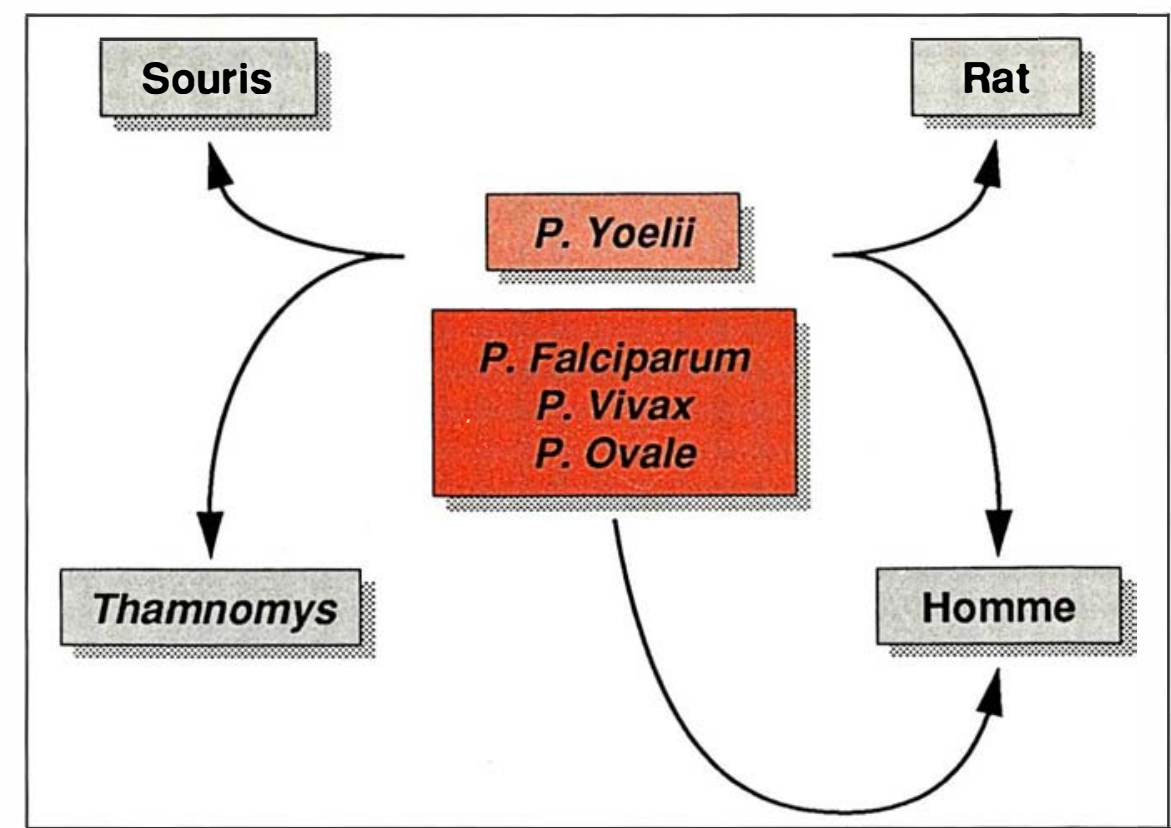

Figure 2. Modèles in vitro utilisés dans notre laboratoire. Le parasite murin P. yoelii se développe "aisément "dans les hépatocytes de souris, de rat, de Thamnomys gazellae, de même que dans des hépatocytes humains. Les plasmodies humaines sont plus exigeantes: P. vivax ne se développe que dans des cellules humaines (hépatome ou primocultures d'hépatocytes) et, jusqu'à présent, seuls les hépatocytes humains permettent la maturation de P. falciparum et de P. ovale. Toutes les plasmodies, y compris les plasmodies humaines, pénètrent in vitro tout type de cellule. Le succès d'une culture dépend donc du profil métabolique de l'hépatocyte et non de la présence d'un récepteur spécifique.

cytes $T$ activés, la première cytokine supposée pouvoir interagir avec la phase hépatique a été l'interféron $\gamma$ (IFN- $\gamma$ ). Des expériences menées in vitro et in vivo, dans des modèles murins et simiens, indiquent que cette cytokine peut efficacement inhiber le développement hépatique du parasite. Une des contributions originales du groupe de Victor Nussenzweig a été de supposer que des sporozoïtes vivants pouvaient induire la sécrétion, par des lymphocytes $T$ sensibilisés, de taux protecteurs d'IFN- $\gamma$ [32]. En effet, l'administration d'anticorps monoclonaux antiIFN- $\gamma$ à des hôtes protégés détruit cette immunité [13]. Des expériences menées in vitro nous ont permis de disséquer la nature et le site d'action de l'IFN- $\gamma$ [33]. L'adjonction de cet IFN- $\gamma$ à des primocultures d'hépato- cytes avant l'inoculation des sporozoïtes inhibe totalement et à très faible dose $(10 \mathrm{U} / \mathrm{ml})$ le développement hépatique du Plasmodium, que l'IFN soit naturel ou recombinant. Sans effet sur le sporozoïte libre, l'IFN- $\gamma$ a une action antiparasitaire par l'intermédiaire de la stimulation de la cellule hôte. In vitro comme in vivo, quelques parasites échappent à l'effet de l'IFN- $\gamma$, de même qu'ils échappent à l'action des anticorps. Dans les deux cas, la différence entre populations susceptible et résistante n'est pas de nature génétique [34]. Dans le cas de l'IFN- $\gamma$, cette résistance pourrait être en relation avec la densité de récepteurs à l'IFN- $\gamma$ à la surface de l'hépatocyte. Il faut de plus souligner que l'effet de l'IFN- $\gamma$ n'est pas limité à un effet parasitostatique, puisque l'addition d'IFN- $\gamma$ 


\section{RÉFÉRENCES}

14. Weiss RW, Sedegah M, Beaudoin RL Miller LH, Good MF. CD8 ${ }^{+} \mathrm{T}$ cells (cytotoxic/suppressors) are required for protection in mice immunized with malaria sporozoites Proc Natl Acad Sci USA 1988; 85 : 573-6.

15. Sadoff JC, Ballou WR, Baron LS, et al. Oral Salmonella typhimurium vaccine expressing circumsporozoite protein protects against malaria. Science 1988 ; 240 : 336-8.

16. Mazier D, Landau I, Druilhe P, et al. Cultivation of the liver forms of Plasmodium vivax in human hepatocytes. Nature 1984 307 : 367-9.

17. Mazier D, Beaudoin RL, Mellouk S, et al. Complete development of hepatic stages of Plasmodium falciparum in vitro. Science $1985 ; 227: 440-2$

18. Mazier D, Collins WE, Mellouk S, et al. Plasmodium ovale: in vitro development of hepatic stages. Exp Parasitol 1987 ; 64 : 393400 .

19. Mazier D, Landau I, Miltgen F, et al. Infestation in vitro d'hépatocytes de Thamnomys adulte par des sporozoïtes de Plasmodium yoelii: schizogonie et libération de mérozoïtes infestants. CR Acad Sci Paris $1982 ; 294$ : 963-5.

20. Mazier D, Mellouk S, Beaudouin RL, et al. Effect of antibodies to recombinant and synthetic peptides on Plasmodium falciparum sporozoites in vitro. Science $1986 ; 231$ : 156-9.

21. Nudelman S, Rénia L, Charoenvit Y, et al. Dual action of anti-sporozoite antibodies in vitro. J Immunol 1989; 143 : 996-1000.

22. Mellouk S, Mazier D, Druilhe P, Berbiguier N, Danis M. In vitro and in vivo results suggest that antisporozoite antibodies do not totally block Plasmodium falciparum sporozoite infectivity. $N$ Engl J Med 1986; 315 648.

23. Rénia L, Miltgen F, Charoenvit Y, et al. Malaria sporozoite penetration: a new approach by double staining. I Immunol $1988 ; 112$ : 201-5.

24. Sibley LD, Weidner E, Krakenbuhl JL. Phagosome acidification blocked by intracellular Toxoplasma gondii. Nature 1985 ; 315 : 416-9.

25. Aley SB, Atkinson CT, Aikawa M, Maloy WL, Hollingdale MR. Ultrastructural localization of Plasmodium falciparum circumsporozoite protein in newly invaded à des cultures de schizontes hépatiques déjà développés induit la lyse d'un nombre significatif de parasites [33]. A côté d'un intérêt fondamental pour la compréhension de l'activité de l'IFN- $\gamma$, nous verrons l'importance d'un tel résultat dans l'optique de phénomènes de cytotoxicité : même exprimé tardivement au cours du développement hépatique, un antigène peut engendrer un stimulus efficace.

Induite par le stade sporozoïte, la sécrétion d'IFN- $\gamma$ l'est également par les stades érythrocytaires : des études longitudinales ont pu montrer que l'IFN- $\gamma$ était présent au cours de l'infestation chez l'homme [35]. La production endogène d'IFN- $\gamma$ pourrait ainsi protéger contre une nouvelle infestation plasmodiale.

- Tumor necrosis factor (TNF). Alors que le rôle de cytokines relarguées par les lymphocytes $T$, tel l'IFN- $\gamma$, était clairement établi, on pouvait imaginer que d'autres cytokines, produites par des monocytes ou des macrophages activés, étaient également impliquées dans la modulation du développement du stade pré-érythrocytaire.

Nous avons donc, in vivo et in vitro, étudié l'effet du TNF (A. Nussler $e t$ al., manuscrit en préparation). In vitro, aucun effet détectable n'est observé avec des concentrations allant de 1 à $5 \mu \mathrm{g} / \mathrm{ml}$ de TNF $\alpha$ recombinant, quel que soit le temps d'incubation. Ces résultats diffèrent de ceux obtenus par Scoffield et al. [36], qui observent que le TNF inhibe le développement de $P$. berghei dans des cellules d'hépatome. Cette inhibition est vraisemblablement liée à l'origine tumorale de la lignée. Nos résultats in vivo sont en accord avec ceux de Scoffield et al. : l'administration intraveineuse de $1 \mu \mathrm{g}$ de TNF interfère avec l'infection sporozoïtaire de $P$. yoelii.

Ces résultats ne nous permettent cependant pas d'exclure une action du TNF au niveau érythrocytaire [37]. Des expériences fondées sur des co-cultures d'hépatocytes et de cellules non parenchymateuses devraient nous permettre de préciser le rôle joué par cette cytokine au niveau hépatique.
- Interleukine-1, interleukine-6 et les facteurs non spécifiques. Rôle de la CRP. L'interleukine-1 (IL-1) inhibe fortement et à dose faible $(5 \mathrm{U} / \mathrm{ml})$ le développement hépatique de $P$. falciparum [33]. Sans effet sur le sporozoïte lui-même, l'IL-1 agit de façon indirecte sur les premières phases de développement. Une des premières hypothèses que nous ayons envisagée est en relation avec la synthèse d'une des protéines de la phase aiguë, la CRP, synthétisée à taux élevé par les hépatocytes stimulés par l'IL-1 [38] et connue pour jouer un rôle non négligeable dans les mécanismes de défense non spécifiques contre les bactéries [39]. Cette hypothèse a été vérifiée dans des modèles in vitro et in vivo [40]. Les tests d'inhibition in vitro ont montré que la CRP inhibait la phase hépatique. Cette activité a été confirmée dans un modèle in vivo : des rats ayant reçu une injection d'huile de térébentine, substance connue pour augmenter de façon significative le taux sérique de CRP [41], se sont montrés réfractaires à l'inoculation de sporozoïtes de $P$. yoelii. Les sporozoïtes préincubés dans de la CRP purifiée perdent en partie leur infectiosité. Il en est de même quand l'incubation a lieu dans des sérums de phase aiguë, les anticorps antiCRP abolissant cette activité antiinfectieuse. La CRP se lie à la surface des sporozoïtes de façon dépendante du calcium et probablement par l'intermédiaire de sites phosphorylcholine. Cette interaction entre CRP et composants de surface du sporozoïte nous avait fait attribuer l'effet inhibiteur à un masquage des sites de reconnaissance entre parasite et cellule cible. L'absence d'inhibition au niveau érythrocytaire en dépit d'une fixation très forte de la CRP sur la forme libre érythrocytaire, le mérozoïte, est en réalité évocatrice de la mise en jeu de mécanismes plus complexes.

Des expériences complémentaires ont été réalisées pour analyser le mode d'action de la CRP purifiée. A un effet sur la pénétration du sporozoïte s'ajoute un effet intracellulaire, responsable du blocage du parasite au stade de trophozoïte, empêchant son passage au stade de schizonte. Ce mécanisme d'inhibition est à rapprocher de l'effet intra- 
cellulaire observé avec les anticorps anti-CS. Comme eux, la CRP liée au parasite pourrait bloquer la translocation de la protéine CS vers la membrane de la vacuole parasitophore, entraînant l'acidification de cette vacuole et la digestion lysosomique du parasite. Cet effet intracellulaire de la CRP souligne l'intérêt potentiel d'une intervention thérapeutique durant cette phase (A. Nussler $e t$ al., soumis pour publication).

Des concentrations élevées de CRP sont retrouvées dans le sérum de malades atteints de paludisme [42]. Le rôle que peut jouer la CRP dans l'acquisition d'une protection antiplasmodiale chez les sujets vivant en zone d'endémie reste à étudier. Il est cependant clair que la CRP en ellemême ne peut résumer tous les effets de l'IL-l et plus généralement les manifestations d'un syndrome inflammatoire. Quand on analyse les rôles respectifs joués par les hépatocytes et/ou le sérum des rats ayant un niveau élevé de protéines de phase aiguë, il apparâit que les facteurs inflammatoires autres que la CRP sont également impliqués, leurs effets inhibiteurs étant observés essentiellement durant la maturation du parasite.

Il a été récemment démontré que l'activité hépatocytaire de l'IL-l impliquait la sécrétion hépatique d'IL-6 [43]. Nous savons maintenant que l'effet de l'IL-l sur le développement hépatique des plasmodies est en partie expliqué par une activité IL-6. Parallèlement à un effet en relation avec le relargage de protéines de phase aiguë, l'IL-6 a un effet direct sur la pénétration et le développement du sporozoïte (S. Pied et al., manuscrit en préparation). On ignore le type cellulaire impliqué dans la sécrétion d'IL-6. Hépatocyte? Autres types cellulaires toujours présents dans une primoculture d'hépatocytes ?

Des taux élevés d'IL-6 ont été retrouvés durant les phases érythrocytaires d'un accès paludique [44]. Cette cytokine pourrait ainsi limiter les surinfections paludiques.

Cytotoxicité : rôle joué par les antigènes parasitaires exprimés à la surface de l'hépatocyte. Il a été suggéré [13, 14] que les lymphocytes suppresseurs/cytotoxiques expri$\mathrm{m} / \mathrm{s} n^{\circ} 10$ vol. 5 , décembre 89 mant le phénotype $\mathrm{CD}^{+}$pourraient participer au développement de la protection induite par les sporozoïtes irradiés. Leur cible en serait non pas les sporozoïtes, formes libres, mais les hépatocytes infectés, capables d'exprimer à leur surface, conjointement les antigènes d'un parasite intracellulaire et des antigènes majeurs d'histocompatibilité (CMH) de classe I [45]. Nous avons utilisé nos modèles in vitro et une panoplie d'anticorps reconnaissant le sporozoïte et/ou les stades hépatiques pour détecter la présence d'épitopes à la surface des hépatocytes infectés. Jusqu'à présent, aucun des anticorps monoclonaux dirigés contre la protéine CS n'a reconnu un antigène à la surface de l'hépatocyte. Il est cependant impossible d'exclure une transformation (processing) des antigènes CS les rendant inaptes à être reconnus par des monoclonaux produits contre l'antigène original. On ne peut de même exclure l'hypothèse que l'antigène CS soit exprimé en quantité suffisante pour induire une réponse cellulaire $T$ mais indétectable par nos techniques. Ces hypothèses sont en accord avec l'obtention de lymphocytes $T$ cytotoxiques chez des souris immunisées avec un épitope cellulaire présomptif de la protéine CS de $P$. yoelii, cela en l'absence de toute détection antigénique sur la membrane hépatocytaire (L. Rénia $e t$ al., manuscrit en préparation).

Contrairement aux antigènes CS, un épitope correspondant au fragment C-terminal de la protéine heat-shocklike de $72 \mathrm{kDa}$, l'hsp 70-l de $P$. falciparum, est exprimé au niveau de la membrane hépatocytaire [46]. Différentes techniques, incluant la microscopie électronique à balayage, ont permis de mettre en évidence, au niveau de l'hépatocyte infecté, un marquage limité à la surface hépatocytaire située au-dessus du parasite. La possibilité pour cet épitope d'engendrer une cytotoxicité restreinte par le CMH est envisagée. Un rôle en relation avec une cytotoxicité induite par les anticorps (ADCC, antibodydependent cell cytotoxicity) a, en revanche, été déjà démontré. Cette activité est indépendante des cellules mononucléées du sang périphérique, mais est partiellement réalisée par les cellules spléniques et surtout par les cellules hépatiques non parenchy- mateuses, puisque l'addition de ces cellules aux cultures hépatiques dans un rapport 2/1 lyse spécifiquement jusqu'à $50 \%$ des schizontes. Si les mécanismes d'ADCC que nous avons observés in vitro existent in vivo, une réponse anticorps induite par un antigène érythrocytaire pourrait agir en rétro-régulation sur le stade antérieur, confirmant le statut privilégié de la phase hépatique.

\section{Phagocytose}

En 1982, Jap et al. mirent en évidence, à proximité de schizontes de $P$. berghei, une réaction locale à base d'infiltrations de cellules mono- et polynucléaires [47]. Des études récentes ont clairement montré que macrophages, polynucléaires neutrophiles et monocytes pouvaient détruire des cellules hépatiques infestées [48]. A l'aide de techniques microcinématographiques, nous avons également observé que des schizontes hépatiques pouvaient être envahis par des filopodes de cellules macrophagiques attirées par le parasite (J. Goma et al., manuscrit en préparation). Ce mécanisme est apparemment en relation avec l'apparition d'un antigène situé dans la vacuole parasitophore de $P$. yoelii. Ce résultat est concordant avec les observations de Meis et Verhave [48], qui décrivent l'ingestion du matériel «spumeux » présent dans la vacuole parasitophore par des polynucléaires neutrophiles. Nous voudrions de plus insister sur le fait que cet antigène est un de ceux que nous avons trouvés exprimés à la surface de l'hépatocyte infesté (Rénia et al., manuscrit en préparation).

\section{Conclusions}

Dans la perspective d'un vaccin antisporozoïte, les anticorps sont restés longtemps les seuls protagonistes dignes de considération. Peu de chercheurs se sont risqués à imaginer le rôle potentiel de mécanismes indépendants des anticorps $[12,49]$. Tomber dans l'excès inverse et n'attribuer, selon la tendance actuelle, aucun intérêt aux anticorps, est aussi déraisonnable. Le fait qu'à faible taux des anticorps puissent induire un effet opposé devrait plutôt stimuler les 


\section{RÉFÉRENCES}

26. Halstead SB, Porterfield JS, O'RourkeEJ. Enhancement of dengue virus infection in monocytes by flavivirus antisera. $\mathrm{Am}$ $J$ Trop Med Hyg 1980; 29 : 638-42.

27. Schartzman JD. Inhibition of a penetration enhancing factor of Toxoplasma gondii by monoclonal antibodies specific for rhoptries. Infect Immunol 1986; 51 : 760-4.

28. Mendis KN, Peiris JSM, Premawansa S, et al. Immune modulation of parasite transmission in Plasmodium vivax malaria. Antigamete antibodies can both block and enhance transmission. In : Molecular Strategies of Parasite invasion. New York: Alan R. Liss, Inc, $1987: 417-26$.

29. Vaughan JA, Do Rosario V, Leland P, et al. Plasmodium falciparum: ingested antisporozoite antibodies affect sporogony in Anopheles stephensi mosquitoes. Exp Parasitol $1988 ; 66$ : 171-82

30. Halstead SB, O'Rourke EJ. Dengue viruses and mononuclear phagocytes. Infection enhancement by non-neutralizing antibody. J Exp Med 1977 ; 146: 201-17.

31. Cardosa JM, Porterfield JS, Gordon S Complement receptor mediates enhancement flavivirus replication in macrophages. $J$ Exp Med 1983 ; 158 : 258-63.

32. Ferreira A, Schofield L, Enea V, et al. Inhibition of development of exoerythrocytic forms of malaria parasites by gamma interferon. Science 1986; $232: 881-4$.

33. Mellouk S, Maheshwari RK, RhodesFeuillette A, et al. Inhibitory activity of interferons and interleukin-l on the development of Plasmodium falciparum in human hepatocyte cultures. J Immunol 1987 ; 139 : 4192 5.

34. Vergara U, Ferreira A, Schellekens $\mathrm{H}$, Nussenzweig V. Mechanisms of escape of exoerythrocytic forms (EEF) of malaria parasites from the inhibitory effects of interferon J Immunol 1987; 138 : 4447-9.

35. Rhodes-Feuillette A, Bellosguardo $M$ Druilhe $\mathrm{P}, e t$ al. The interferon compartment of the immune response in human malaria. II. Presence of serum interferon-gamma following the acute attack. I Interferon Res $1985 ; 5$ : 169-74.

36. Schofield L, Nussenzweig RS, Nussenzweig V. CD8 ${ }^{+}$T-cells and gamma-interferon required for immunity to sporozoite challenge. Report of the tenth meeting of the scientific working group on the immunology

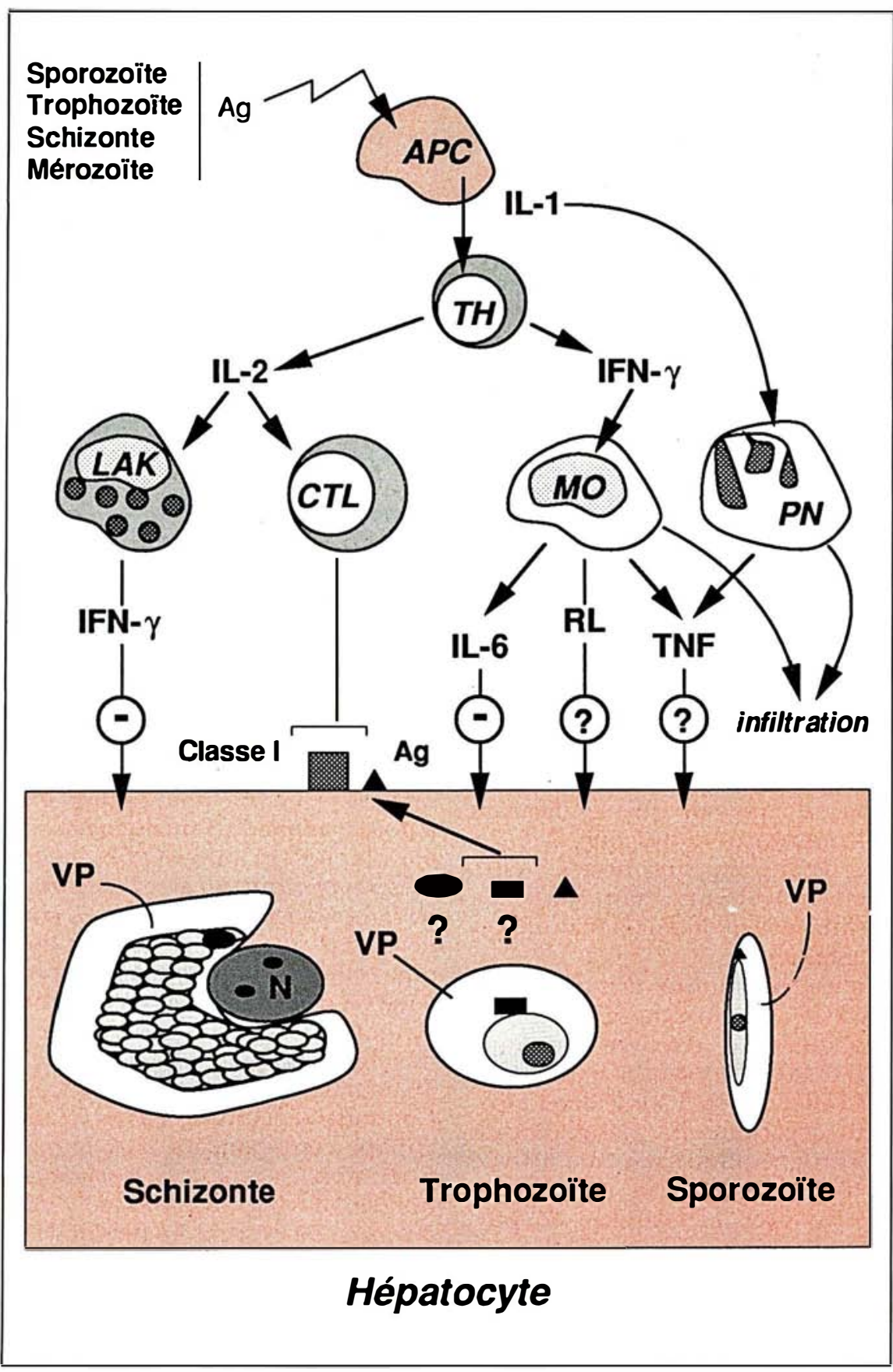

Figure 3. Immunité cellulaire. L'IL-1 relarguée par les cellules présentant l'antigène (APC) active les cellules polynucléaires (PN) et les cellules $T$ auxiliaires (TH) qui vont sécréter IL-2 et interféron (IFN- $\gamma$ ). L'IL-2 active les lymphokineactivated killer cells (LAK) et les cellules cytotoxiques (CTL), tandis que I'IFN- $\gamma$ active les macrophages (MO) qui à leur tour vont sécréter de I'IL-6. Un certain nombre de mécanismes interfèrent avec le développement hépatique du Plasmodium: phagocytose consécutive à une infiltration par les polynucléaires et les macrophages; Iyse et/ou inhibition du parasite par I'IFN- $\gamma$. Contrairement à I'IL-1, I'IL6 a un effet direct sur le développement du parasite. Le rôle des radicaux libres (RL) est inconnu, l'efficacité du tumor necrosis factor (TNF) est controversée. Quant aux CTL, nous avons pu mettre en évidence un effet cytotoxique dirigé contre le stade hépatique, effet induit par un épitope de la région non répétée de la protéine CS de P. yoelii. VP = vacuole parasitophore $N=$ noyau de l'hépatocyte. 


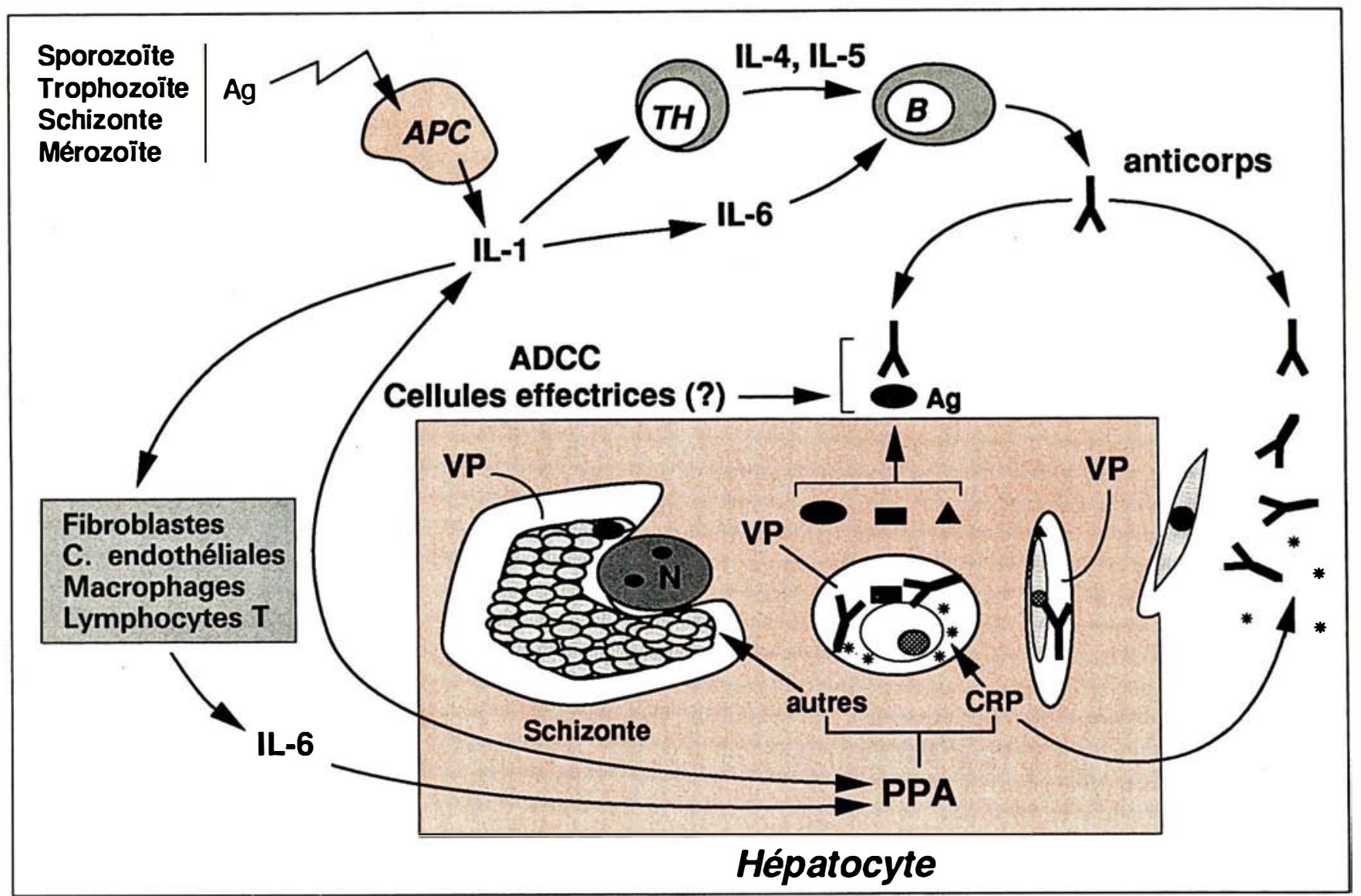

Figure 4. Immunité humorale. La transformation (processing) des antigènes plasmodiaux (Ag) par les cellules présentant l'antigène (APC) conduit à la sécrétion d'll-1 et d'IL-6. Ces deux Iymphokines agissent sur la phase hépatique de deux manières: (1) une cascade d'activations donne lieu à la synthèse d'anticorps. Les anticorps anti-sporozoïte préviennent l'attachement du sporozoïte et/ou sa pénétration. Quand ces anticorps sont internalisés dans la vacuole parasitophore NP), ils entraînent l'arrêt du développement du trophozoïte, souvent même sa disparition. Les anticorps dirigés contre le stade hépatique sont également inhibiteurs. Certains d'entre eux agissent de plus dans le cadre de mécanismes de coopération cellulaire: des antigènes du parasite intra-hépatique, exprimés à la surface de l'hépatocyte sont, au moins in vitro, la cible de mécanismes de cytotoxicité dépendante des anticorps $(A D C C)$; (2) I'IL-1 induit la synthèse de protéines de la phase aiguë (PPA) par les hépatocytes, soit directement, soit par l'intermédiaire d'IL-6. Parmi les différentes protéines inflammatoires, la protéine C-réactive (CRP) inhibe le développement initial du sporozoïte. Nous savons maintenant qu'un mécanisme mimant l'action des anticorps et inhibant la translocation de la protéine CS à la membrane de la vacuole parasitophore est également imputable à la CRP. Contrairement à la CRP, la majorité des autres protéines de phase aiguë agissent tardivement, sur la maturation du schizonte hépatique. $\mathrm{TH}=$ cellule $T$ auxiliaire (helper); $B=$ lymphocyte $B ; A D C C=$ cytotoxicité dépendante des anticorps; $N=$ noyau de l'hépatocyte.

recherches concernant la régulation de leur apparition ainsi que leur mode d'action.

Les figures 3 et 4 cherchent à résumer le champ très mouvant des interactions hôte-parasite au niveau préérythrocytaire. Elles illustrent le statut privilégié du parasite en phase hépatique, phase modulable, non $m / s n^{\circ} 10$ vol. 5 , décembre 89 seulement par le sporozoïte, mais également par le stade érythrocytaire. De nombreux points d'interrogations persistent. Leur solution en soulèvera vraisemblablement de nouveaux. Par la multiplicité et la complexité des mécanismes effecteurs interagissant à son niveau, la phase hépatique séduit de plus en plus de chercheurs. Les possibilités d'intervention sur ces mécanismes en justifient l'intérêt. Nous devons cependant garder à l'esprit que ce parasite, non seulement sait déjouer des mécanismes effecteurs très puissants, mais qu'il est de plus capable de les utiliser pour son propre développement 


\section{RÉFÉRENCES}

37. Taverne J, Tavernier J, Fiers W, Playfair JHL. Recombinant tumour necrosis factor inhibits malaria parasites in vivo but not in vitro. Clin Exp Immunol 1987; 67 : 1-4.

38. Ramadori G, Sipe JD, Dinarello GA, Orizel SBB, Colten HR. Pretranslational modulation of acute phase hepatic protein synthesis by murine recombinant interleukin1 (IL-1) and purified IL-l. J Exp Med 1985 ; $162: 930-42$.

39. Mold C, Rodgers PC, Kaplan R, Gewurtz $\mathrm{H}$. Binding of human $\mathrm{C}$-reactive protein to bacteria. Infect Immun $1982 ; 38$ : 392 -

40. Pied S, Nussler A, Pontet M, et al. C-reactive protein protects against pre-erythrocytic stages of malaria. Infect Immun 1989; 57 : 278-82.

41. Kushner I, Feldmann G. Control of the acute phase reponse. Demonstration of Crelative protein synthesis and secretion by hepatocytes during acute inflammation in the rabbit. J Exp Med 1978 ; 22 : 466-77.

42. Naik P, Voller A. Serum C-reactive protein levels and falciparum malaria. Trans $R$ Soc Trop Med Hyg 1984 ; 78: 812-815.

43. Helle M, Brakenhoff JP, De Groot ER, Aarden LA. Interleukin-6 is involved in interleukin-l induced activities. Eur J Immunol $1988 ; 18: 957-9$.

44. Grau GE, Frey K, Fontana A, et al. Modulation of IL-6 production in experimental cerebral malaria. Berlin:7th International Congress of Immunology, 1989.

45. Germain RN. Ins and outs of antigen processing and presentation. Nature 1986; $322: 687-9$.

46. Rénia L, Mattei D, Goma J, et al. A mala ria heat shock like protein epitope expressed on the infected hepatocyte surface is the target of ADCC mechanisms. Pre-erytrocytic stage malaria vaccine development : current status and future prospects. Bethesda : Naval Medical Research Institute 1989; 1-51.

47. Jap PHK, Meis JFGM, Verhave JP, Meuwissen JHET. Degenerating exoerythrocytic forms of Plasmodium berghei in rat liver : an ultrastructural and cytochemical study. Parasitol $1982 ; 85$ : 263-9.

48. Meis JFGM, Verhave JP. Exoerythrocytic development of malaria parasites. Adv Para sitol $1988 ; 27$ : 1-66.

49. Spitalny GL, Verhave JP, Meuwis sen JHETH, Nussenzweig RS. Plasmodium berghei: T-cell dependence of sporozoiteinduced immunity. Exp Parasitol 1977; 42 :

\section{Summary}

Protective role of the hepatic phase of malaria

Protection against pre-erythrocytic stages of malaria is possible as demonstrated by the generation of resistance after immunization with irradiated sporozoites. The objective of an antisporozoite vaccine would be to mimic this strong protective response. However, the mechanisms involved are more numerous and intricate than previously believed and it progressively appears that the role of the presumed target, the sporozoite, might be negligible compared to that of the hepatic stage. Moreover, recent results tend to demonstrate that interdependences with the ensuing erythrocytic stage might also exist. Thus, besides its potential interest as a target in itself, the hepatic stage of malaria might play a crucial role as "go-between " with other stages. The previous and the subsequent developmental stages can modulate the «shielded» phase by cytokines, directly or as a result of a cascade of events, by antibodies, and by MHC-restricted or antibody dependent cytotoxic mechanisms.

\section{Remerciements}

Les recherches du laboratoire sont en partie financées par des contrats OMS (UNDP. /World Bank/Who Special Programme on Training and Research in Tropical Diseases), la Commission des Communautés européennes et le Conseil scientifique Pitié-Salpêtrière.

\section{TIRÉS A PART}

D. Mazier. 